\title{
CALCULATIONS OF UNSTEADY FLOWS AROUND HIGH-LIFT CONFIGURATIONS BASED ON A ZONAL APPROACH
}

\author{
S. Bosnyakov, I. Kursakov, S. Mikhaylov, and V. Vlasenko \\ Central Aerohydrodynamic Institute (TsAGI) \\ 1 Zhukovsky Str., Zhukovsky, Moscow Region 140180, Russia
}

\begin{abstract}
Zonal approach for unsteady Reynolds-averaged Navier-Stokes (URANS) problem solution is described. Original feature of this approach is to use Courant-Friedrichs-Levi number CFL $\sim 1$ in the main part of calculation domain excluding thin part of boundary layer. It is achieved by using explicit numerical scheme with fractional time stepping in the main part of calculation domain. In the near-wall zone of boundary layer, implicit dual stepping method is used. In addition to zonal approach, fully implicit method with dual stepping technology is also implemented. The methods are verified in comparison with the results of test case data obtained by consortium participants within DeSiReH FP-7 project.
\end{abstract}

\section{INTRODUCTION}

The work has been done in the framework of the DeSiReH Project, Task 1.3. $\mathrm{DeSiReH}$ is a European project on design, simulation and flight Reynolds number testing for advanced high-lift solutions. Its objective is to improve the industrial design process for high lift devices for laminar wings in terms of product quality, efficiency, and development cost reduction. The objective of Task 1.3 is acceleration of unsteady computations in an optimization process. So, the purpose of this work is to increase the efficiency of numerical solution of nonstationary problem about wing with released high-lift devices in turbulent flow of real gas at critical and supercritical incidence angles. Nonstationary processes have to be described with adequate quality.

The flow near wing with released high-lift devices has a rather complicated structure and an interaction of many physical effects. It is essentially viscous flow, where developed turbulent boundary layers generate the flow structure. In spite of small Mach numbers $(\mathrm{M} \sim 0.2)$, which correspond to takeoff and landing regimes, supersonic zones may be present in flow around strongly deflected slat.

This is an Open Access article distributed under the terms of the Creative Commons Attribution License 4.0, which permits unrestricted use, distribution, and reproduction in any medium, provided the original work is properly cited. 
In the case of medium incidence angles, the time-averaged flow is stationary and can be simulated numerically with the use of present methods. But there are some problems with correct prediction of drag and lift coefficients of wing. But more serious troubles arise in the case of large incidence angles, when, because of stall, there are nonstationary processes connected with the strong interaction of developed separation zones with the nonstationary vortex sheet past the wing.

Since the flow is nonstationary, one should choose explicit schemes for simulation. These schemes permit to describe nonstationary processes with high quality. But the other peculiarity of such tasks is the problem of multiscale: characteristic times and sizes of different physical processes can differ for some orders of values. Therefore, using explicit schemes leads to extremely large calculation time. In the case of multiscale problems, implicit schemes are good; nevertheless, they have very poor quality for description of nonstationary processes.

A possible way out of this contradiction is to use the zonal method. In this method, flow zones with very small scales of physical processes (mainly, inner zones of boundary layers) are calculated using the implicit scheme, while the other part of flow is calculated using the explicit one. As a result, the nonstationary processes in inviscid core of flow are simulated with high quality. In the inner part of boundary layers, an implicit scheme is used and one may hope for good results, because the information has to be transmitted across the boundary layer; and the nonstationary processes in the inner zone of boundary layer have, mainly, to conform to main nonstationary processes that take place in inviscid core of flow. This consideration reduces scheme requirements, from the viewpoint of nonstationary process description quality, and permits to use the implicit scheme in such concrete zones.

\section{METHOD}

All the calculations were performed based on the full three-dimensional (3D) nonstationary Reynolds equation system closed by Menter shear stress transport (SST) turbulence model. The basic variant of solver, which had been developed by authors previously, is implemented based on the finite-volume numerical method that has the second approximation order in all variables and includes the monotonic modified Godunov scheme for approximation of convective fluxes, the central-difference approximation of diffusive fluxes, and two-layer point-implicit approximation of source terms. Detailed description of this method is given in [1]. The calculations are performed on multiblock structured grid with hexahedral cells. The method permits to use irregular joining the blocks with the discontinuity of grid lines at the boundaries of blocks.

The explicit schemes are the best choice for some nonstationary applications, when the time scale is comparable with the ration between space scale and maximal eigenvalue, i.e., when Courant number defined by physics is about one. For example, it takes place in aeroacoustics, direct numerical simulation (DNS), and 
large-eddy simulation (LES). For such applications, the explicit Runge-Kutta scheme is popular enough. But in case of problems with large and medium characteristic time scales and with very strong discrepancy of cell sizes, the restriction because of stability can be many orders less that the characteristic physical time of flow changes. It is the problem that is under consideration in this work, i.e., the nonstationary phenomena connected with the flow separation at the wing in case of large incidence angles (stall). In such a case, the calculation with global CFL about hundreds or even thousands can be performed without simulation accuracy alleviation. In this work, another ideology is supposed. The problem is formulated to achieve a high-quality but computationally efficient numerical simulation of nonstationary processes at the wing with high-lift device at large incidence angles for takeoff and landing regimes. For this purpose, the rather fast calculations that can be included (partly, at least) into engineering process of aerodynamic design are necessary. Therefore, an inevitable choice in thin near-wall zone of boundary layer (where maximal possible time step, from the viewpoint of explicit scheme stability, is for some orders less than characteristic time of nonstationary processes in considered problem) is to use the "time-accurate" implicit scheme with dual-time stepping. But in the other part of flow, the calculation should be performed with Courant number being about one for high-quality description of nonstationary processes, even in the case of implicit scheme. In this case, the "time-accurate" implicit scheme has unacceptably high computer costs. Therefore, it is assumed to use the explicit scheme in the other part of solution. At that, a fractional time stepping method is used to accelerate the calculation with explicit scheme. This method permits to keep the high quality of nonstationary process description.

Thus, the computational domain is subdivided into two zones (each of them can be topologically multiply connected). In the first zone (that includes the near-wall zones of boundary layers), the calculation is performed using the implicit scheme with dual-time stepping. In the second one (that contains the other part of computational domain), the calculation is performed using the explicit scheme with fractional time stepping. This method will be named later on as a Zonal Decomposition approach and the description of this numerical method composition (an explicit scheme with fractional time stepping and an implicit scheme with dual-time stepping) will be done.

Bellow, for simplicity and brevity, the schemes will be formulated for a scalar model equation that contains convective fluxes only:

$$
\frac{\partial u}{\partial t}+\frac{\partial F(u)}{\partial x}=0 \text {. }
$$

To speed up calculations of steady flows, the implicit scheme with global time stepping is used. Such schemes may be considered as standard for calculations of stationary flows. For scalar model (1), this scheme may be represented in the following form: 


$$
\begin{aligned}
\frac{u_{i}^{n+1}-u_{i}^{n}}{\tau}+\frac{A_{i+1 / 2}\left[\tilde{U}_{i+1 / 2}^{n+1}-\tilde{U}_{i+1 / 2}^{n}\right]}{}-A_{i-1 / 2}\left[\tilde{U}_{i-1 / 2}^{n+1}-\tilde{U}_{i-1 / 2}^{n}\right] & h \\
+ & \frac{F_{i+1 / 2}\left(u^{n}\right)-F_{i-1 / 2}\left(u^{n}\right)}{h}=0 .
\end{aligned}
$$

In this scheme, both the approximation of the physical time derivative $\partial u / \partial t$ and the implicit part of spatial operator has only the first accuracy order. Jacoby matrices $A_{i+1 / 2}=A\left(u_{i}^{n}, u_{i+1}^{n}\right)$ are calculated at the known time layer $n$. Only the explicit part of spatial operator is approximated using the 2nd-accuracy order scheme.

The system of algebraic linear equations, which is based on Eq. (2), is solved approximately by 6 iterations of Gauss-Seidel method for block-diagonal matrices. In the beginning of computation, the value of global time step $\tau$ is not very large, but it is increased gradually, tending to the given value of $\mathrm{CFL}_{\max }$. If some problems with stability arise, the time step is diminished temporarily; when the stability returns, the step begins to grow again. It is obvious that such an approach excludes any possibility of correct simulation of nonstationary phenomena. If the local value of Courant number CFL $\gg 1$, one cannot even speak about the approximation of the derivative $\partial u / \partial t$ : the errors of approximation may be higher than the approximated derivative itself. The spatial operator is not approximated either because of the linearization of nonlinear fluxes. Incomplete Gauss-Seidel iterations crown this list of errors. From the other side, each global time step is performed very quickly (only several times slower than one global time step with explicit scheme) and the scheme is generally stable; if the stationary solution is achieved, it provides the required 2nd-accuracy order in space, because the stationary limit of Eq. (2) coincides with the stationary limit for the explicit scheme. If the stationary solution exists, the convergence to this stationary solution is usually better and essentially quicker than the convergence for explicit scheme with local-time stepping or with multigrid acceleration. So, this scheme is a very good choice for stationary tasks solution. But within the current work, the unsteady phenomena only are of interest.

A dual-time stepping method is usually used for realization of time-accurate implicit scheme instead of traditional methods for solving the large nonlinear algebraic equation systems. In case of such an approach, the 2nd-order scheme in time is used, and a fictitious nonstationary term is added:

$$
\begin{aligned}
\frac{\partial u}{\partial \xi}+\left(1+\frac{\tau_{n}}{\tau_{n-1}+\tau_{n}}\right) \frac{u-u_{i}^{n}}{\tau_{n}}-\frac{\tau_{n}}{\tau_{n-1}+\tau_{n}} \frac{u_{i}^{n}-u_{i}^{n-1}}{\tau_{n-1}} & \\
+ & +\frac{F_{i+1 / 2}(u)-F_{i-1 / 2}(u)}{h_{i}}=0 .
\end{aligned}
$$

Here, $\xi$ is the pseudotime. The stationary solution of the problem (3), when it is single, coincides with the solution of the discrete equation without pseudotime 
term: in the stationary limit, $u=u^{n+1}$. Because it is time-marching method and one is interested in a stationary solution only, different methods of convergence acceleration may be used to obtain this solution. The high-efficient implicit scheme with strongly simplified implicit operator like Eq. (2) is used in this work:

$$
\begin{aligned}
& u_{i}^{(0)}=u_{i}^{n} ; \\
& \frac{u_{i}^{(k+1)}-u_{i}^{(k)}}{\Delta \xi}+\frac{3}{2} \frac{u_{i}^{(k+1)}-u_{i}^{n}}{\tau}-\frac{1}{2} \frac{u_{i}^{n}-u_{i}^{n-1}}{\tau} \\
& +\frac{A_{i+1 / 2}\left[\tilde{U}_{i+1 / 2}^{(k+1)}-\tilde{U}_{i+1 / 2}^{(k)}\right]-A_{i-1 / 2}\left[\tilde{U}_{i-1 / 2}^{(k+1)}-\tilde{U}_{i-1 / 2}^{(k)}\right]}{h} \\
& +\frac{F_{i+1 / 2}\left(u^{(k)}\right)-F_{i-1 / 2}\left(u^{(k)}\right)}{h}=0, k=1, \ldots, M ; \\
& u_{i}^{n+1}=u_{i}^{(M)} .
\end{aligned}
$$

Such a scheme has only the first order in pseudotime that results both in the calculation acceleration and in the improvement of its stability.

To speed-up calculations in inviscid core, a method of fractional time stepping is used. The idea of fractional time stepping is that the calculation in each cell is performed with the larger time step (i.e., with maximal possible Courant number). But the numbers of interim time steps are different in different cells and they are chosen so as all the cells achieve the same layer of physical time in some moments. It should be noted that the first description of such a method that is known by authors of the current work was given in [2].

As Eq. (1) describes the information transfer over space with velocity $d x / d t$ $=a(u)$, the information transfers per one time step the distance $\mathrm{CFL}_{i} h_{i}$ where $\mathrm{CFL}_{i}=a_{i} \tau_{n} / h_{i}$ is the local Courant number. A standard approach for time stepping organization (global time stepping) is the following. The most rigid condition for time step $\left(\tau_{\min }=\min _{i} \tau_{i}^{\text {stab }}\right)$ is chosen and time step $\tau_{n}=\tau_{\min }$ is performed in all cells. Let it be that $\tau_{\min } \ll \tau_{\max }=\max _{i} \tau_{i}^{\text {stab }}$. It is typical for strongly nonuniform grids that are used for simulation of flows with boundary layers. At that time, the Courant number $\mathrm{CFL}_{i}=a_{i} \tau_{\min } / h_{i}=\tau_{\min } / \tau_{i}^{\text {stab }} \ll 1$ in most cells. Then, the information in major part of cells passes only small part of grid step $h_{i}$ per time step. Therefore, the information propagates very slowly over the computational domain and a lot of time steps are necessary to describe the characteristic interval of global flow changing. The idea of fractional time stepping is that the calculation in each cell is performed with the highest time step (i.e., with maximal possible Courant number). But the numbers of local time steps are different in different cells and they are chosen so as all the 


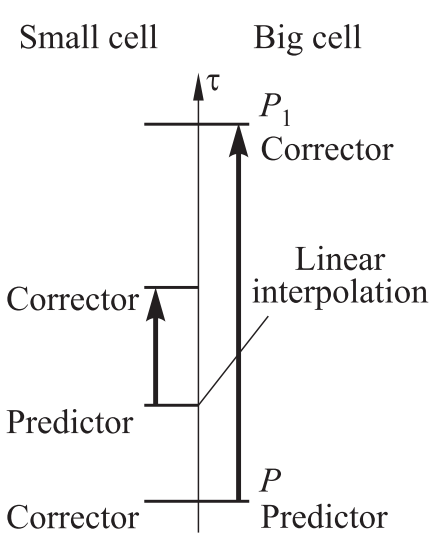

(a)

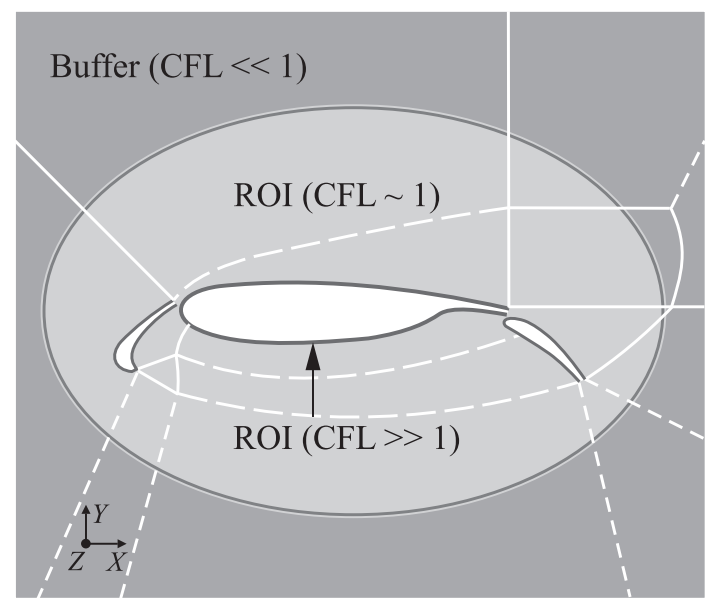

(b)

Figure 1 Description of fractional time stepping

cells achieve the same layer of physical time in some moments (Fig. 1a). When the same time layer is achieved, let name it as a completion of global time step. For example, if an interim time step in the cell $A$ is equal to $\tau_{\max }$, in $B-$ to $\tau_{\max } / 2$, and in $C-$ to $\tau_{\max } / 8$, then, during one global time step, one should perform one interim time step in cell $A$, two interim time steps in cell $B$, and eight interim time steps in cell $C$. Therefore, the global time step in each cell is divided (fragmented) into smaller interim time steps so as the interim time steps satisfy the local restriction on time step in the given cell. That is why the procedure is named as fractional time stepping.

Let consider the fractional time stepping organization in details. Before beginning of the calculation, the maximal possible value of time step is determined in each cell of computational domain. Then, the maximal time step in the whole computational domain $\left(\tau_{\max }=\max \tau_{i}^{\text {stab }}\right)$ is determined. Then, the value of interim time step is determined in each cell. The time step value in the cell with the number $i$ is taken equal to $\tau_{i}=\tau_{\max } / 2^{l}$. The integer-valued parameter $l$ is chosen so as the local stability condition $\left(0.5 \tau_{i}^{\text {stab }}<\tau_{i} \leq \tau_{i}^{\text {stab }}\right)$ is satisfied in the given cell. As a result of this procedure, the whole collection of computational domain cells is divided into some subsets that will be named as levels. In all cells of $m$-level, the value of interim time step is equal to $\tau_{i}=\tau_{\max } / 2^{M-m}$ where $M$ is the number of levels. In the cells of the first level, the interim time step is minimal: it is equal to $\tau_{i}=\tau_{\max }$ in the cells of $M$-level.

Let emphasize that, because the time step in each cell is defined by local time-dependent conditions of flow, the procedure of dividing the cells into the 
levels is performed before each global time step. During one global time step, $2^{M-m}$ interim time steps are performed in the cell of $m$-level. The number of interim time steps is different in different cells. But towards the end of the global time step, time in all cells increases by the same value $-\tau_{\max }$. As a result, the nonstationary development of flow is described correctly. This procedure diminishes the total calculation time in rational programming (because few time steps are performed in large cells) and guaranties that the local value of stability coefficient $\left(C_{\text {stab }} \in[0.5 ; 1]\right)$ is used in each cell. In organization of fractional time stepping procedure, it is very important to synchronize the steps in different cells. Let consider, for example, a case, when there are three groups of cells. The value of interim time step in the first group is equal to $\tau_{\max }$, in the second one - to $\tau_{\max } / 2$, and in the third one - to $\tau_{\max } / 4$. Thus, if total number of levels of cells is equal to $M$, then the quantity of passes through the computational domain during one global step is $2 \cdot 2^{M-1}=2^{M}$. At that, the calculations during one pass are not performed in all cells - only in the cells of some levels.

The fractional time stepping is very easy to implement. An almost complete set of formulas needed to implement the fractional time stepping in source code with global time stepping is as follows:

$-\tau_{\min }-$ minimal time step;

- $\tau_{\text {cell }}$ - time step at the cell;

- $n_{\text {cell }} \geq \tau_{\text {cell }} / \tau_{\text {min }}-$ fractional steps number in the cell;

$-n_{\max }=\max _{\substack{i \\ 6}}\left(n_{\text {cell }}\right)$

$-n_{\text {inter }}=\min _{i}^{6}\left(n_{\text {cell }} / 2\right)$ - interpolation step;

$-\left(n+n_{\text {cell }}\right) \%\left(2 n_{\text {cell }}\right)=0$ - predictor condition;

$-n \%\left(2 n_{\text {cell }}\right)=0-$ corrector condition;

$-n \% n_{\text {inter }}=0$;

$-d n=\left(n_{\max }-n\right) / n_{\text {inter }}$ - interpolation condition; and

- $P=\left(P_{1}+P d n\right) /(d n+1)$ - interpolation value.

However, the greater the number of levels $M$, the less is the computation efficiency. Thus, if the aspect ratio of the maximum to the minimum cell is 10 , the step time is to increase by 10 times too, and the average number of interim steps and, accordingly, the computation time of global step increase only 1.5 times. For the aspect ratio of $10^{3}$, the time of calculation of the global step increases 10 times. This is acceptable. However, for the aspect ratio of $10^{6}$ (the turbulent boundary layer calculations), the calculation of the global step increases 1000 times, making the calculations actually impossible. That is why the computational domain has been divided into three zones (Fig. 1b). The boundary layer region is calculated with CFL $\gg 1$ with the dual-time stepping 
implicit scheme. An area in the vicinity of the wing (Region Of Interest ROI) is calculated with CFL $\sim 1$ using the explicit scheme with fractional time stepping. It is here that the accuracy of the unsteady processes simulation is maximized. The buffering area far away from the wing is calculated by the explicit scheme with fractional time stepping too, but $\mathrm{CFL} \ll 1$.

\section{TESTS FOR EUROPIV2 AIRFOIL}

For the development of methodology, it is natural to use two-dimensional (2D) test task, because it requires essentially less great computer resources and allows parametric calculations for the comparison of various variants. For this purpose, the test task based on EUROPIV2 experimental data has been chosen. The experiment had been performed in the Low Speed Wind Tunnel of Airbus Bremen (Germany) in October $2002[3,4]$. The straight wing (span - $2.1 \mathrm{~m}$ ) with three-element airfoil RA16SC1 had been tested. The slat and flap angles were set at constant position with deflection angles of $30^{\circ}$ and $40^{\circ}$, respectively. The reference chord (that corresponds to retracted slat and flap) is equal to $c$ $=0.5 \mathrm{~m}$. During the tests, the wind-tunnel free-stream speed was equal to $54 \mathrm{~m} / \mathrm{s}(\mathrm{M}=0.15)$ under atmospheric conditions. The Reynolds number, based on the free-stream velocity and on the chord length of the main wing, was equal to $1.7 \cdot 10^{6}$. Measurements included both the registration of the static pressure distributions and the mapping of the velocity fields by particle image velocimetry (PIV) method.

The implementation of Zonal Decomposition approach requires using the multiblock computational grid with a special structure. Each solid body within the computational domain should be surrounded by a thin layer of the grid blocks, where the implicit scheme with dual-time stepping will be applied. The grid, which has been constructed for simulation of EUROPIV2 tests, is shown in Fig. 2. The grid consists of 67,008 cells and is subdivided into 31 blocks. Around the airfoil main element, the width of the near-wall layers is about $0.2 \mathrm{~mm}$ that is about $3 \%$ of boundary layer thickness at the point with maximal $y$-coordinate on the upper surface of the airfoil. The width of the near-wall layers near the flap and the slat was chosen to be 2 times lower - about $0.1 \mathrm{~mm}$. The total number of cells in boundary blocks was equal to 21,120 . It is worth to point to the irregular connection of the near-wall and the outer blocks of grid: the grid lines are not continuous on the boundaries between the near-wall and the outer blocks.

Calculations have been performed for the following series of the angle of attack: $\alpha=5^{\circ}, 9^{\circ}, 12^{\circ}, 17.5^{\circ}, 19^{\circ}$, and $24^{\circ}$. Figure 3 shows the structure of flow around airfoil for some values of $\alpha$.

For the incidence angle $\alpha=5^{\circ}$, one may see three recirculation zones in flow around the airfoil. One zone is placed below the slat. It is formed in the 


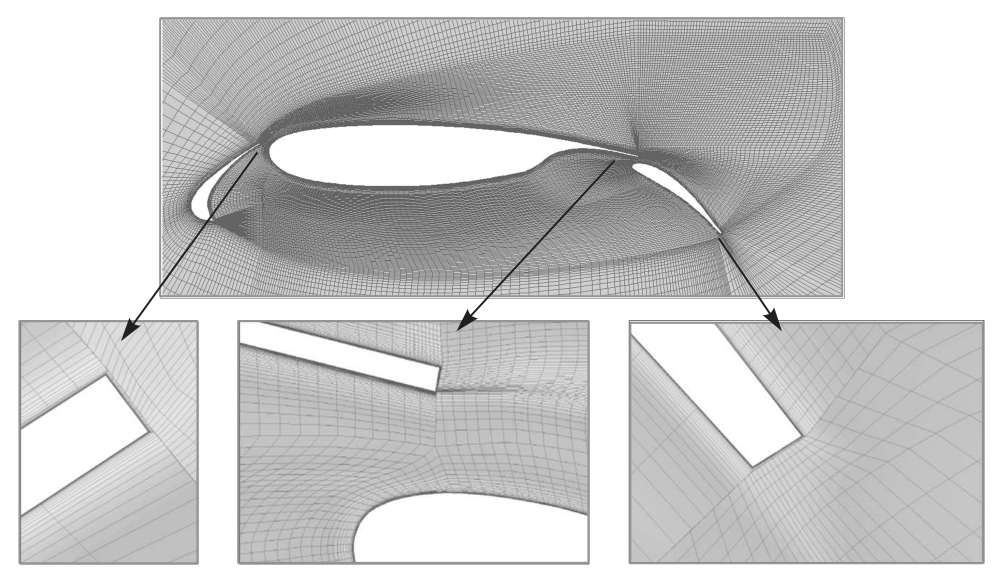

Figure 2 Computational grid around EUROPIV2 airfoil

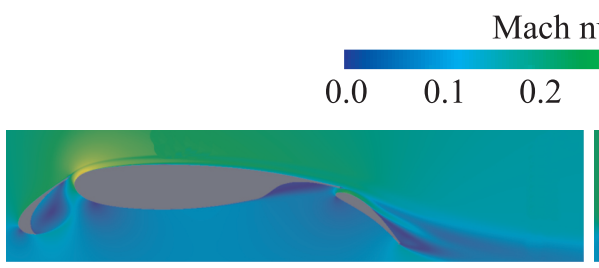

(a)

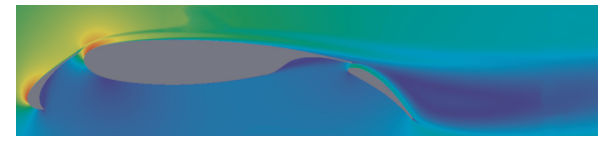

(c)

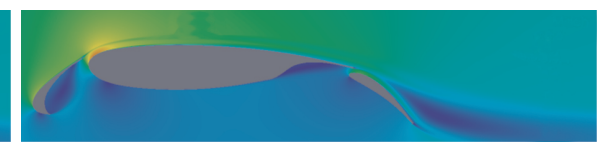

(b)

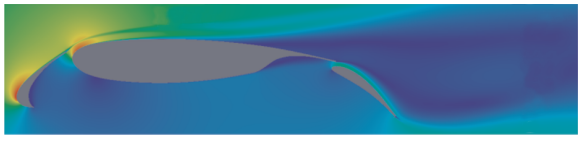

(d)

Figure 3 Flow structure around the airfoil: $(a) \alpha=5^{\circ} ;(b) 9^{\circ} ;(c) 19^{\circ}$; and (d) $\alpha=24^{\circ}$

stagnation region past the sharp lower edge of the slat. The second recirculation zone is formed below the main element of the airfoil, where the flap is retracted. The boundary layer on the upper surfaces of the slat and the flap is laminar. The flow comes off from the flap trailing edge at the higher angle with respect to the flap surface. This causes the deceleration of flow near the flap trailing edge. As a result, the laminar boundary layer on the flap upper surface separates from the flap with formation of the third recirculation zone. The flow inside all three recirculation zones is turbulent. Behind the airfoil main element, the boundary layer becomes turbulent around the first sixth of the chord. Downstream from 
the trailing edge of the airfoil main element, one may see the turbulent shear layer that is produced by the mixing of flow behind the airfoil with the gas that passes through the space between the main element and the flap. Starting conditions for this mixing layer are formed by the turbulent boundary layer on the main element upper surface, so that this mixing layer is turbulent immediately from the trailing edge. The analogous mixing layer is formed downstream from the slat; it is also turbulent from the beginning due to the turbulence in the recirculation zone below the slat. This mixing layer merges with another mixing layer downstream from the main element. Behind the flap, this joint mixing layer turns up with deceleration of flow (and the pressure grows).

At the incidence angle $\alpha=9^{\circ}$, the recirculation zones behind the slat and the main element become smaller and the turbulence in these zones becomes weaker. The separation behind the flap practically disappears (one may find separation just near the very trailing edge). The turbulence in joint mixing layer behind the flap is higher; because of the disappearance of boundary layer separation, this mixing layer has to turn more sharply in front of the flap trailing edge; it leads to stronger deceleration of flow in this layer.

At the incidence angle $\alpha=19^{\circ}$, the recirculation zones, behind the slat, practically disappear. In the Mach number field, one may find the trace of mixing layer past the slat, with diminishing of Mach number, produced by growth of turbulence in this mixing layer and by the corresponding transition of the main flow kinetic energy into the kinetic energy of turbulence. There is no separation on the flap upper surface. But in the stagnation region near the joint mixing layer inclination in front of the flap trailing edge, the vortex is formed.

Finally, at the incidence angle $\alpha=24^{\circ}$, one may see the stall-like phenomenon - the flow separation from the airfoil main element upper surface. The large vortex is formed in stagnation zone behind the flow separation; it interacts with the vortex behind the flap.

In Fig. 4, the computed distributions of pressure coefficient $C_{p}$ over the airfoil surface are shown for some values of $\alpha$. Because the airfoil was calculated in conditions of infinite flow, for the comparison with EUROPIV2 experiment, it is necessary to correct the incidence angle in order to take into account the influence of the wind tunnel walls. According to [4], the flow picture around the airfoil in infinite flow at $\alpha=9^{\circ}$ should be analogous to flow picture around airfoil inside the wind tunnel at $\alpha \approx 10.5^{\circ} ; \alpha=12^{\circ}$ in infinite flow corresponds to $\alpha \approx 14.5^{\circ}$ in the wind tunnel, and so on. For $\alpha=5^{\circ}, 9^{\circ}$, and $12^{\circ}$, the computed distributions are compared with the closest experimental curves. One may see that the maximal similarity to the dependence $C_{p}(x)$, obtained in computation for $\alpha=9^{\circ}$, was achieved in experiment at $\alpha \approx 12^{\circ}$ (which is between $\alpha \approx 10.5^{\circ}$ and $\alpha \approx 14.5^{\circ}$, indeed). The maximal similarity to the dependence $C_{p}(x)$, obtained in computation for $\alpha=12^{\circ}$, was achieved in experiment at $\alpha \approx 16^{\circ}$. The comparison of computed and experimental dependences $C_{p}(x)$ for all three incidence angles shows that the rarefaction above the flap is underestimated and 

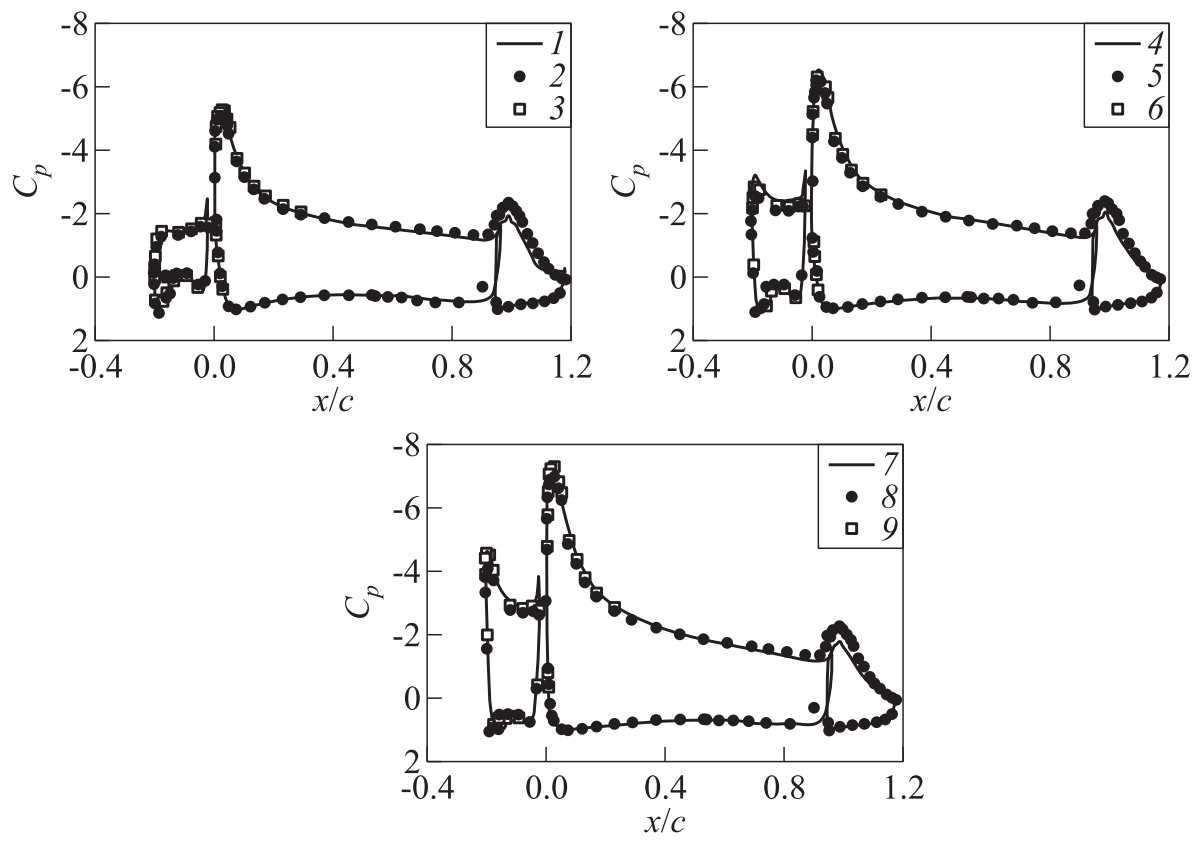

Figure 4 Distributions of pressure coefficient over the airfoil: $1-\alpha=5^{\circ} ; 2-5^{\circ}$ (interpolated between $\alpha=4^{\circ}$ and $6^{\circ}$ ); $3-6^{\circ} ; 4-9^{\circ}, 5-11^{\circ}, 6-12^{\circ} ; 7-12^{\circ}$, $8-15^{\circ}$, and $9-\alpha=16^{\circ}$. Curves refer to calculations and signs refer to experiments

the pressure in the flap retraction region is overestimated. Some discrepancies may also be found for the slat upper surface.

In Fig. 5, the fields of horizontal and vertical components of velocity, obtained by PIV method within the EUROPIV2 Project for the incidence angle $\alpha=12^{\circ}$, are compared with the analogous fields, obtained in computation for the incidence angle $\alpha=9^{\circ}$. At the first sight, these fields are very similar; however, the experimental picture shows the separation on the flap upper surface; on the contrary, this separation is practically absent in the computation. Accordingly, the flow deceleration above the flap is greater in computation because of the more sharp turn of the flow in front of the flap trailing edge. It may be assumed that the computation overestimates the turbulence intensity in the boundary layer on the upper surface of the airfoil main element and in the following mixing layer. Due to this overestimation, the time-averaged mixing layer in computation becomes more stable and presses the flow towards the flap upper surface, preventing the separation and increasing the pressure. The overestimation of pressure in the flap retraction region near the main element trailing edge may also be explained by this phenomenon. 


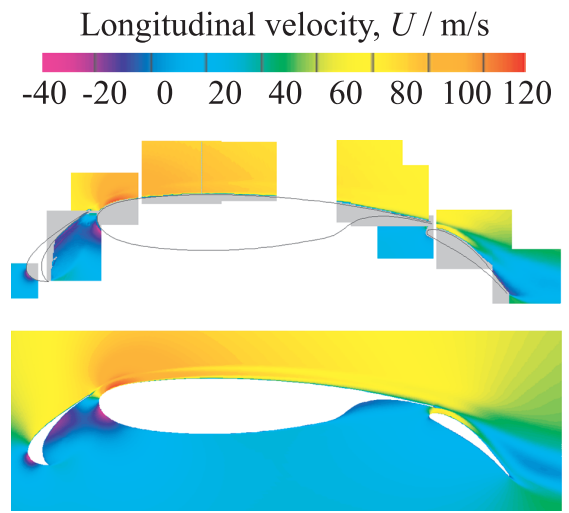

(a)

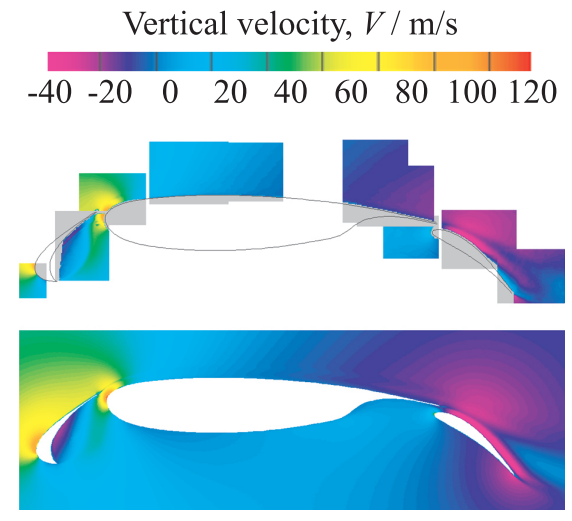

(b)

Figure 5 Comparison of PIV-measured velocity fields (upper plots) with computations (lower plots): (a) longitudinal velocity $U$; and $(b)$ vertical velocity $V$

To choose the optimal variant of numerical methodology for calculation of flows around wing at high angles of attack, the additional computations of flow around the EUROPIV2 airfoil have been performed for one high value of the incidence angle: $\alpha=19^{\circ}$. The following variants of numerical method have been considered:

- Explicit scheme with local time stepping (hereunder, this scheme will be named as "local explicit") — standard way to accelerate convergence to stationary solution, if the explicit numerical scheme is used. In each cell, the local value of time step $\tau_{n}$ corresponded to $\mathrm{CFL}=0.95$;

- Implicit scheme (2) with global time stepping (hereunder this scheme will be named as "global implicit"). Three variants of $\mathrm{CFL}_{\max }$ are compared: $\mathrm{CFL}_{\max }=10^{4}, 10^{6}$, and $10^{10}$. The $\mathrm{CFL}_{\max }=10^{4}$ variant leads to $\mathrm{CFL} \leq 1$ in ROI. In the case $\mathrm{CFL}_{\max }=10^{6}$, one has the local values of Courant number in ROI CFL $\sim 100$, and the $\mathrm{CFL}_{\max }=10^{10}$ version results in CFL $\sim 10^{6}$ in ROI. This should result in principally incorrect description of essentially unsteady process, but some hope still remains that the time-averaged solution would still be similar to reality;

- Implicit scheme (4) with dual-time stepping (hereunder, this scheme will be named as "dual implicit"). This scheme has nominally 2ndapproximation order in physical time (and first order in pseudotime). Calculations were performed with constant step in physical time $\tau_{n}$; the following variants were considered: $\tau_{n}=5 \cdot 10^{-3}, 5 \cdot 10^{-4}, 5 \cdot 10^{-5}$, and $5 \cdot 10^{-6} \mathrm{~s}$. 
In outer part of boundary layers, CFL $\leq 1$ only when $\tau_{n}=5 \cdot 10^{-6} \mathrm{~s}$ (and even then CFL $>10$ in the vicinity of trailing edges of the airfoil elements). For $\tau_{n}=5 \cdot 10^{-5} \mathrm{~s}, \mathrm{CFL} \in[10 ; 20]$ in outer part of boundary layers, and the unsteady processes are described with high inaccuracy here. One can hope only that the 2 nd order of accuracy nominally will allow describing the main features of unsteady processes. For $\tau_{n}=5 \cdot 10^{-4} \mathrm{~s}$, CFL $>100$ in the outer part of boundary layers, and for $\tau_{n}=5 \cdot 10^{-3} \mathrm{~s}$, CFL $>1000$ in the same area. So, for such values of $\tau_{n}$, the description of essentially unsteady process should be incorrect, and one may only hope that nevertheless, the time-averaged solution will be similar to reality; and

- Zonal decomposition method (hereunder, this scheme will be named as "zonal"). In this case, the calculation has been performed with the constant global step in physical time $\tau_{n}=10^{-4} \mathrm{~s}$. In near-wall layer of blocks, the calculation has been performed using the implicit scheme (4) with dual-time stepping $\left(\mathrm{CFL}_{\xi}=10^{10}, N_{\xi}=20\right)$. In ROI, the explicit scheme with fractional time stepping was used. In each cell, the value of local step in physical time corresponded to CFL $\in[0.5 ; 1]$.

Figure 6 shows the behavior of coefficients $C_{p x}, C_{f x}$, and $C_{p y}$, obtained using the various schemes, during the last 5000 iterations of each computation. It is easy to see that the explicit scheme with local time stepping ("local explicit," curves 1) is not applicable to solution of this problem. The local time stepping is reasonable only when the pseudounsteady process converges to the stationary state. But Fig. 6 shows that the local time stepping procedure does not converge in this problem. Instead, it comes to long-period oscillations with large amplitude. This oscillation process is of no physical reason. It is essential that even the average level of these oscillations does not coincide with the average level that is obtained using other numerical approaches.

"Global implicit" schemes with $\mathrm{CFL}_{\max }=10^{6}$ and $10^{10}$ give also incorrect results (curves 3 and 2 in Fig. 6). With $\mathrm{CFL}_{\max }=10^{10}$, one comes also to oscillations with very large amplitude around the wrong average level. In case $\mathrm{CFL}_{\max }=10^{6}$, the amplitude of oscillations is lower, and the average level is another, but it is wrong as well. On the contrary, the "global implicit" scheme with $\mathrm{CFL}_{\max }=10^{4}$ (curves 4 ) converges to the quasi-stationary state with low oscillations around the average level that is very similar to that one predicted by the "zonal" method (curves 9 ).

As for "dual implicit" (curves 5-8) and "zonal" methods (curves 9), all of them give the relatively similar results (in the scale of vertical axis in Fig. 6). Even the "dual implicit" schemes with $\tau_{n}=5 \cdot 10^{-3}$ and $5 \cdot 10^{-4} \mathrm{~s}$ (in ROI, the latter variant is similar to the "global implicit" scheme with $\mathrm{CFL}_{\max }=10^{6}$, and the former is worse) came to the quasi-stationary state that is essentially better than the final state of calculation by the "global implicit" scheme with $\mathrm{CFL}_{\max }=10^{6}$. This result is possible to be explained: the "dual implicit" 

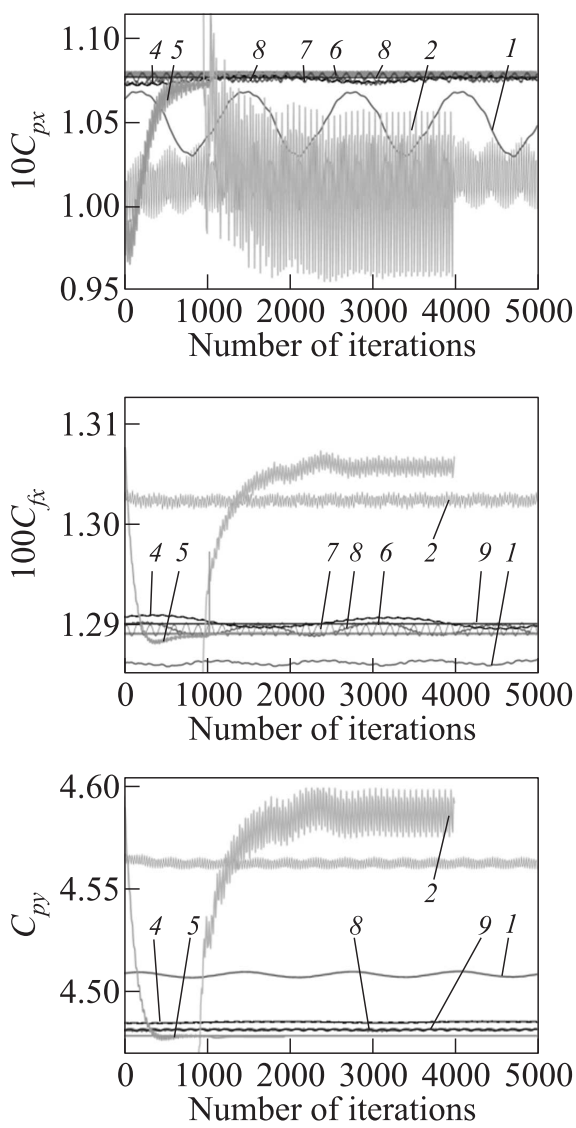

Figure 6 Dependences of integral force coefficients upon the number of iterations for the last 5000 iterations; $\alpha=19^{\circ}: 1-$ local explicit; $2-\mathrm{CFL}_{\max }=10^{10} ; 3-$ $10^{6} ; 4-\mathrm{CFL}_{\max }=10^{4} ; 5-\tau_{n}=5$ $\times 10^{-3} \mathrm{~s}, \mathrm{CFL}_{\xi}=10^{10}, N_{\xi}=20 ; 6-$ $\tau_{n}=5 \cdot 10^{-4} \mathrm{~s}, \mathrm{CFL}_{\xi}=10^{10}, N_{\xi}=20$; $7-\tau_{n}=5 \cdot 10^{-5} \mathrm{~s}, \mathrm{CFL}_{\xi}=10^{10}, N_{\xi}$ $=20 ; 8-\tau_{n}=5 \cdot 10^{-6} \mathrm{~s}, \mathrm{CFL}_{\xi}$ $=10^{10}, N_{\xi}=20 ;$ and $9-$ zonal, $\tau_{n}$ $=10^{-4} \mathrm{~s}$. Dashed curves indicate global implicit and dotted curves indicate dual implicit schemes
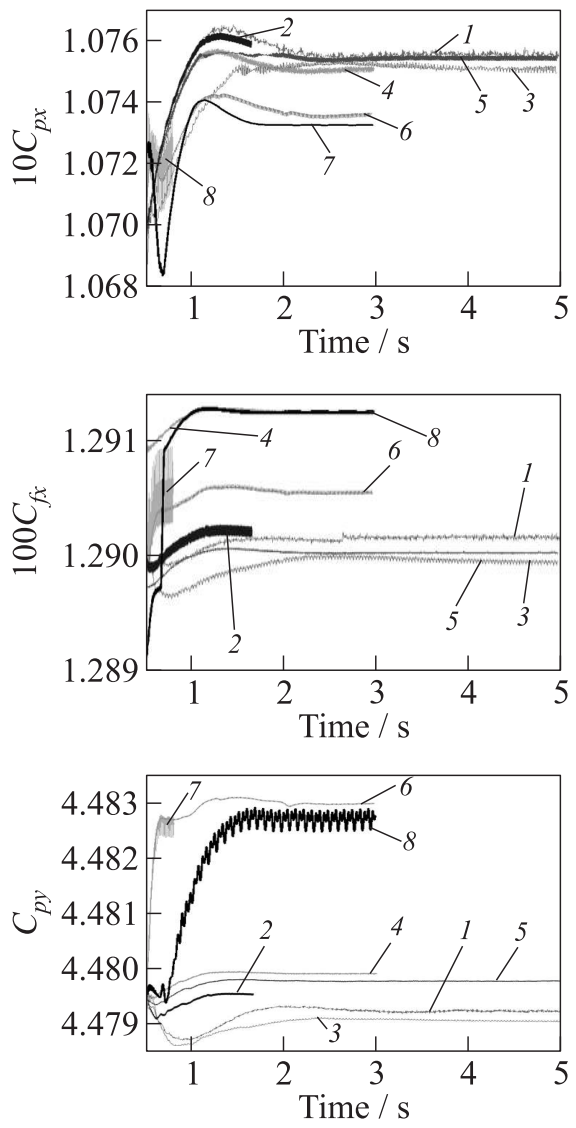

Figure 7 Dependences of integral force coefficients upon time; $\alpha=19^{\circ}: 1-$ $\tau_{n}=5 \cdot 10^{-3} \mathrm{~s}, \mathrm{CFL}_{\xi}=10^{10}, N_{\xi}=20$; $2-\tau_{n}=5 \cdot 10^{-4} \mathrm{~s}, \mathrm{CFL}_{\xi}=10^{4}, N_{\xi}$ $=500 ; 3-\tau_{n}=5 \cdot 10^{-4} \mathrm{~s}, \mathrm{CFL}_{\xi}=10^{4}$, $N_{\xi}=100 ; 4-\tau_{n}=5 \cdot 10^{-4} \mathrm{~s}, \mathrm{CFL}_{\xi}$ $=10^{10}, N_{\xi}=100 ; 5-\tau_{n}=5 \cdot 10^{-4} \mathrm{~s}$, $\mathrm{CFL}_{\xi}=10^{10}, N_{\xi}=20 ; 6-\tau_{n}=5$ $\times 10^{-5} \mathrm{~s}, \mathrm{CFL}_{\xi}=10^{10}, N_{\xi}=20 ; 7-$ $\tau_{n}=5 \cdot 10^{-6} \mathrm{~s}, \mathrm{CFL}_{\xi}=10^{10}, N_{\xi}=20$; and $8-\tau_{n}=10^{-4}$ s. Curves $1-7$ indicate dual implicit and 8 indicates zonal schemes 
scheme uses 20 pseudotime iterations per each physical time step, and each of these pseudotime iterations consists of 6 Gauss-Seidel iterations. This means that the total quantity of iterations for solution of the equation system (4) is $6 \cdot 20=120$ that is essentially better than 6 iterations for solution of system (2) that are used in the "global implicit" scheme. Moreover, the equation system (3) does not contain the linearization of fluxes and nominally has the 2nd-accuracy order in time that should give the essentially better representation of unsteady processes.

Figure 6 compares the schemes, some of which do not describe unsteady processes at all (as the "local explicit" scheme) or describe them with too large errors (as the "global implicit" scheme with $\mathrm{CFL}_{\max } \geq 10^{6}$ ). That is why the coefficients $C_{p x}, C_{f x}$, and $C_{p y}$ are plotted as functions of iteration. To compare the schemes that pretend to more-or-less correct description of unsteady processes (various variants of "dual implicit" schemes and "zonal" method), the coefficients $C_{p x}, C_{f x}$, and $C_{p y}$ can be plotted as functions of physical time. This is made in Fig. 7. It should be noted that all the curves in Fig. 7 are smoothed using the running average procedure.

Dependences $C_{p x}(t)$ and $C_{p y}(t)$ may clearly be subdivided into two groups, which are different in average level of the limit cycle. The "Dual implicit" schemes with $\tau_{n}=5 \cdot 10^{-5}$ and $5 \cdot 10^{-6} \mathrm{~s}$ belong to the same group as the "zonal" method; all other variants converge to the limit cycle with another average level. It agrees with the assumption that the requirement of CFL $\leq 10$ in ROI is essential for the correct representation of unsteady processes - and even of the average levels of the limit cycles of these unsteady processes. Though the other group of curves has no clear physical reason, nevertheless, it is possible to be used for the choice of pseudotime stepping parameters in the "dual implicit" scheme. The dependences $C_{p x}(t)$ and $C_{p y}(t)$ are in agreement with the main assumption that the calculation with high CFL number can be performed in the depth of the boundary layers, but the condition CFL $\sim 1$ should be satisfied in the ROI. From this viewpoint, among all variants, only the "zonal" method and as well as the "dual implicit" calculations with $\tau_{n}=5 \cdot 10^{-5}$ and $5 \cdot 10^{-6} \mathrm{~s}$ can be used to describe the unsteady process; and the "dual implicit" calculation with $\tau_{n}=5 \cdot 10^{-6} \mathrm{~s}$ is more preferable than the "dual implicit" one with $\tau_{n}=5 \cdot 10^{-5} \mathrm{~s}$. The "Zonal" method is doubtless more reliable than the other two: in boundary

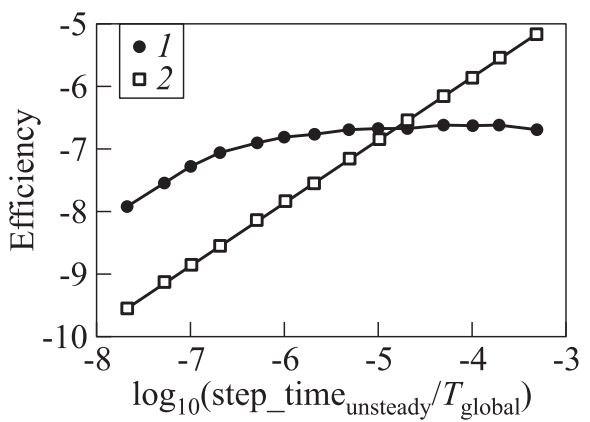

Figure 8 Computational efficiency of zonal (1) approach for airfoil: 2 - dual implicit scheme 
blocks, it coincides with "dual implicit" methods, but in ROI, it exploits the fast and time-accurate fractional time stepping, which is based on the explicit calculation with local CFL $\in[0.5 ; 1]$, without any iterations. Now the computational efficiency of this method is to be estimated (Fig. 8).

The efficiency of "zonal" approach vs. the efficiency of "dual implicit" scheme is shown in Fig. 8.

Along the horizontal axis, the parameter $\log _{10}\left(\right.$ step_time $\left._{\text {unsteady }} / T_{\text {global }}\right)$ is plotted where $T_{\text {global }}$ is the CPU time.

Figure 8 shows that the "zonal" method becomes more efficient than the "dual" one at step_time unsteady $<10^{-4.8} \approx 1.6 \cdot 10^{-5}$ s. Accordingly, the "dual" calculation with step_time unsteady $=5 \cdot 10^{-5} \mathrm{~s}$ runs in physical time faster than the "zonal" method, and only in case step_time ${ }_{\text {unsteady }}=5 \cdot 10^{-6} \mathrm{~s}$, the "zonal" method becomes more efficient (approximately 3 times faster) than the "dual implicit" calculation.

\section{CALCULATIONS OF "WING-FUSELAGE" MODEL}

To verify the robustness and the efficiency of the chosen numerical approach for solution of real 3D problems, the test based on experiments in European transonic wind tunnel (ETW, Cologne, Germany) has been chosen in the framework of EUROLIFT II project $[5,6]$. In this project, the model tested has name as TC217. The KH3Y three-element wing that has a slat and a flap along the wingspan entirely has been used.

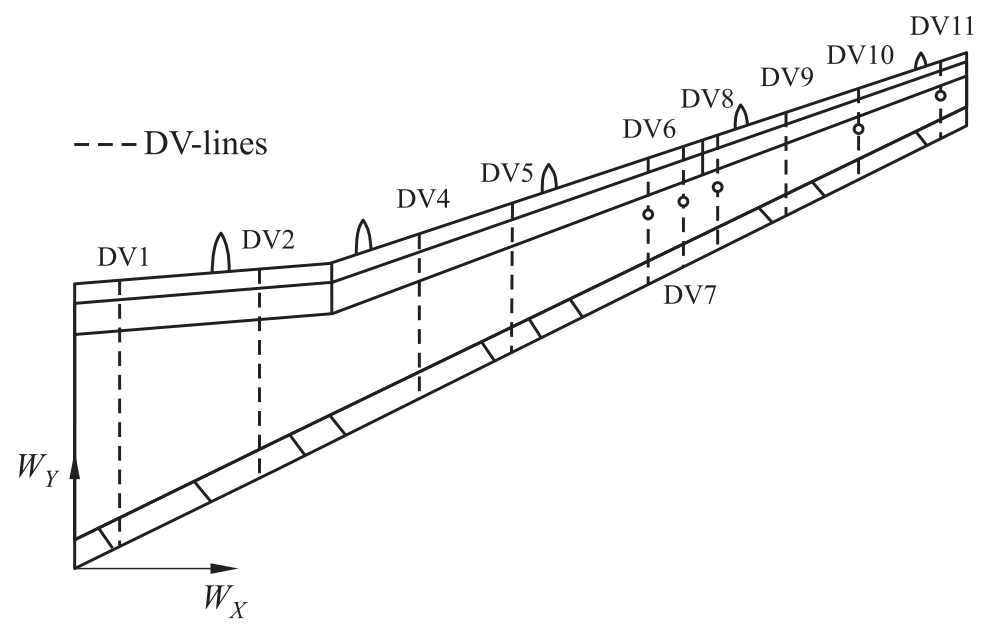

Figure 9 The TC217 model wing and the sections, where the pressure distributions were measured: 1 - DV-lines 


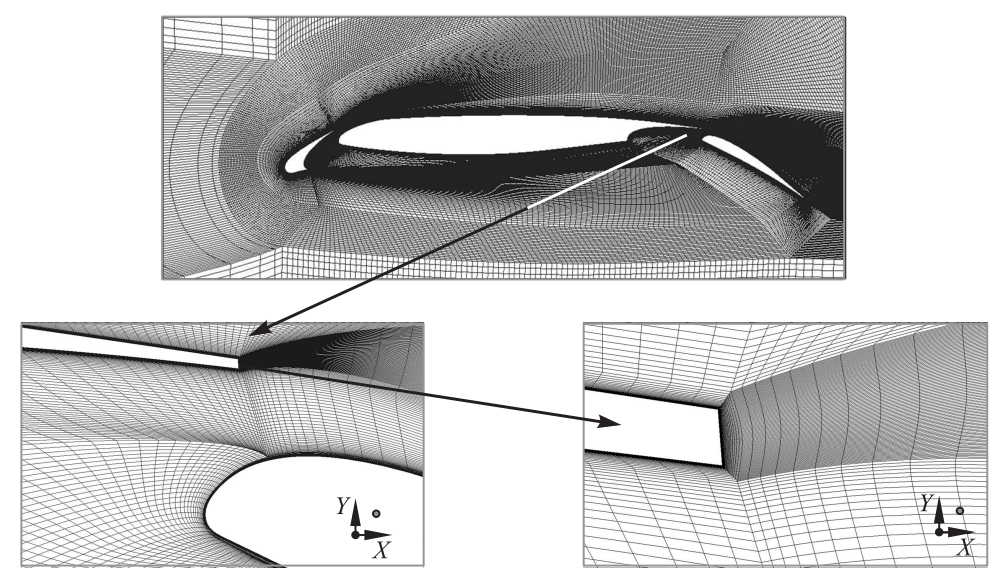

Figure 10 Calculation grid near wing TC217

During the experiment, the integral forces over the model (forces and moments) and the pressure distributions were measured in 10 sections (Fig. 9). It is to be noted that there were no nonstationary measurements. That is why, the numerical methodology developed in the framework of the current work has to obtain the correct time-averaged data and, above all, has to determine correctly a critical incidence angle, when a flow separation from the upper surface of wing (stall) takes place, and the time-averaged characteristics of wing near this critical incidence angle. The polar 239 has been chosen for verification. In this experiment, the Mach number of outer flow was equal to $\mathrm{M}=0.2$ and the Reynolds number based on the averaged chord of wing $\operatorname{Re}=15 \cdot 10^{6}$. A configuration that is characteristic for landing regimes (slat and flap angles were $26^{\circ}$ and $32^{\circ}$, respectively) was chosen. The polar 239 includes the experimental data for incidence angles $\alpha=0.12^{\circ}, 5.05^{\circ}, 10.07^{\circ}, 15.12^{\circ}, 18.02^{\circ}, 19.01^{\circ}, 20.02^{\circ}$, and $21.04^{\circ}$.

As the model incidence angle was equal to zero, the calculation grid was generated for a half model only (half a fuselage + wing). The grid consists of 272 blocks and 16 million cells.

Figure 10 shows the grid in ROI - near wing. In zonal approach, the calculation in the near-wall block layer is performed using the implicit scheme with dual-time stepping. The thickness of this layer is constant everywhere at the main element of wing and equal to $0.2 \mathrm{~mm}$ and it is constant at the slat and the flap and equal to $0.1 \mathrm{~mm}$. The grid across this layer contains 32 cells and the center of the first near-wall cells is situated at distance $y^{+} \leq 1$ from the surface (except the zone near the stagnation point). Two grids were generated "mesh1" (2 million cells) and "mesh2" (0.25 million cells) in addition to this the 
most detailed grid, which contains 16 million cells and hereunder will be named as "mesh0." Each of these two grids is made by removing each second node from the more detailed grid. No-slip boundary condition with zero heat fluxes is formulated for all solid surfaces. The soft boundary condition based on Riemann invariant analysis is formulated for the outer boundary of computational domain.

Figure 11 presents the charts of distributions of pressure coefficient $C_{p}$ over the surface wing for incidence angle $\alpha=21^{\circ}$. These charts were obtained for the grids "mesh0," "mesh1," and "mesh2" in three sections: DV2, DV5, and DV10. The experimental pressure distributions, which were obtained in ETW (Cologne, Germany), are imposed on these charts too. The incidence angle $\alpha=21^{\circ}$ is of interest because in the experiment, at this incidence angle, the stall on the upper surface of the wing tip begins. The distributions $C_{p}(x)$ show that in the case of regimes without separation, the grids "mesh1" and "mesh0" give very similar results. In case of the coarsest grid ("mesh2"), the peaks of distributions $C_{p}(x)$ are smoothed and rarefaction over the upper surface of wing is underestimated. It is interesting, however, that available experimental data (for incidence angle $\alpha=21^{\circ}$ ) are located between the curves obtained using the grids "mesh0" and "mesh1." Above the flap, the coarse grid ("mesh2") corresponds essentially better to the experiment. Among all the grids, the separation begins at $\alpha=21^{\circ}$ (in the wing tip) in case of the coarsest grid ("mesh2"). The curve obtained for the grid "mesh2" is in good correspondence with the experiment data in separation zone. The further calculations at the incidence angle $\alpha=24^{\circ}$ show that the flow separation already propagates up to wing middle in the calculation using the grid "mesh2." In the case of the grid "mesh1," it begins at the wing tip and in case of the calculation using the grid "mesh0," there is still no separation. In case of incidence angle $\alpha=27^{\circ}$, the flow separation reaches the section DV2 in the calculation using the grid "mesh2." In the calculation using the grid "mesh1," it reaches the wing middle. The calculation using the most detailed grid for this incidence angle shows an irregular behavior - the flow separation emerges in the wing middle, while the flow at the wing root and tip remains without separation.

The results obtained can be easy explained, if one takes into account that the least Courant numbers in ROI are achieved for the coarsest grid ("mesh2") among three grids; CFL is two times higher for the grid "mesh1" and it is even four times higher for the grid "mesh0" (Fig. 12). Therefore, from the viewpoint of describing the nonstationary processes (that inevitable arise during the flow separation), the grid "mesh2" is to give the most reliable results. Indeed, only in case of this grid, the flow separation begins appropriately at the same time as in the experiment and in the same place - at the wing end. In the case of grid "mesh1," the flow separation begins in the correct place but it begins later - at $\alpha=24^{\circ}$ (Fig. 13). Finally, in the case of the most detailed grid, the flow separation begins later and in incorrect place. This confirms the 


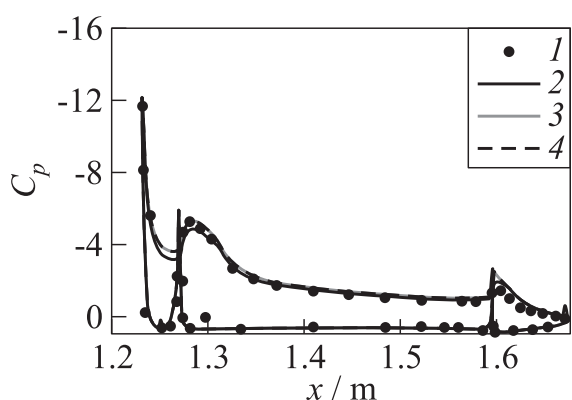

(a)

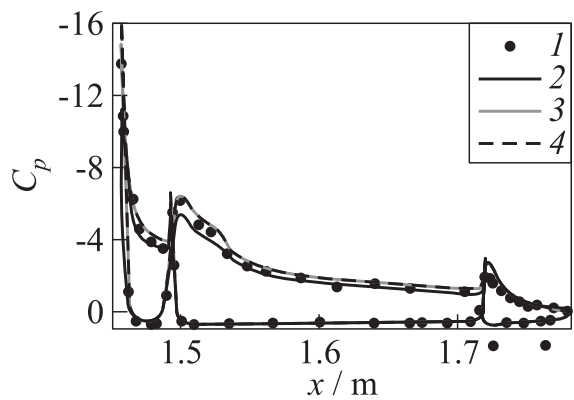

(b)

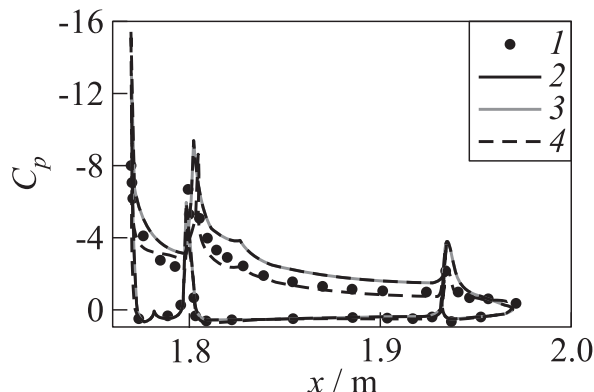

(c)

Figure 11 Distributions of pressure coefficient in sections DV2 $(a)$, DV5 $(b)$, and DV10 $(c)$; incidence angle $\alpha=21^{\circ}: 1$ experiment; 2 - mesh2; 3 - mesh1; and $4-\operatorname{mesh} 0$

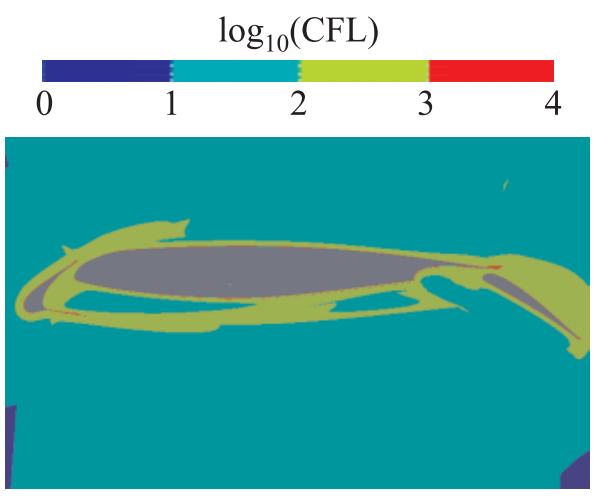

(a)

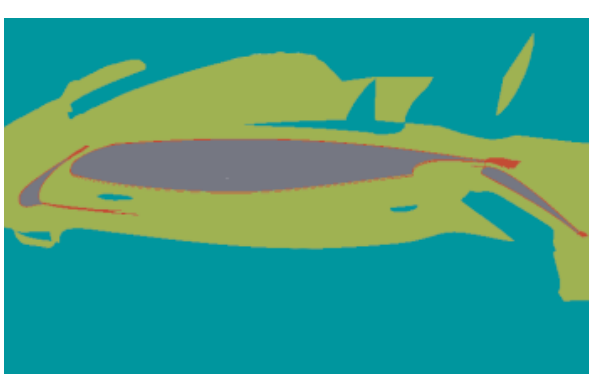

(b)

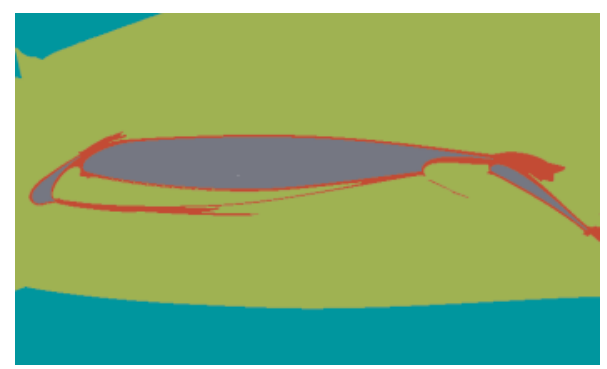

(c)

Figure 12 Fields of Courant number decimal logarithm: (a) mesh2; (b) mesh1; and $(c)$ mesh0 

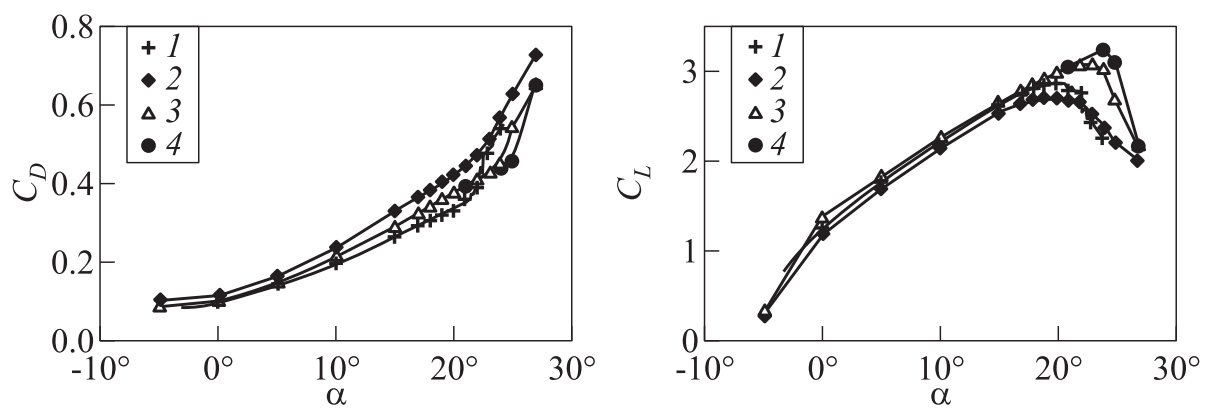

Figure 13 Comparison of calculations using different grids: 1 - experiment; $2-$ mesh2; 3 - mesh1; and $4-\operatorname{mesh} 0$
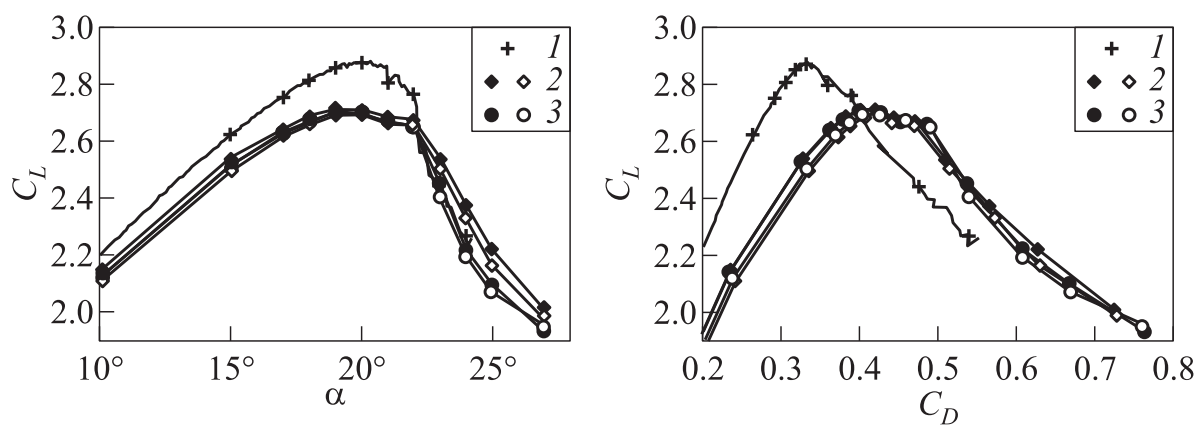

Figure 14 The "dual" scheme (filled signs) and the zonal approach (empty signs) calculations (mesh2) vs. the experiment (1): $2-\tau_{n}=5 \cdot 10^{-4} \mathrm{~s}$; and $3-\tau_{n}=5 \cdot 10^{-6} \mathrm{~s}$

importance of keeping the condition CFL $\sim 1$ (in ROI) in solving the problem under consideration.

The results of calculation are presented in Fig. 14 where charts $C_{D}(\alpha)$ and $C_{L}(\alpha)$ obtained using different schemes are presented. The charts in noseparation zone of flow are practically coincident; therefore, the data for incidence angles that are more than $10^{\circ}$ are presented. It is easy to see that in case of similar time steps, the curves obtained using the "dual" scheme and the zonal approach are close to each other. In diminishing the global physical time step, there is a calculation data approaching tendency to the experimental curve. In case of "dual" scheme, such an improvement of solution is achieved at the high computation cost: the calculation time increases 100 times. For the zonal approach, the calculation time does not change practically.

Calculations of unsteady flows around the high-lift configurations can be performed with high Courant numbers at the depth of boundary layers, if the condition $\mathrm{Cu} \sim 1$ is satisfied in the outer part of the boundary layer and in 
the zone of inviscid flow. In this case, the proposed zonal approach permits to accelerate the description of the nonstationary processes in comparison with the standard approach that are based on using the implicit scheme with dual-time step. For the TC217 model, the acceleration is 2 times higher and more (Fig. 15).

The zonal approach and the dual time stepping provide the same quality of calculations around highlift configurations if the Courant number is the same.

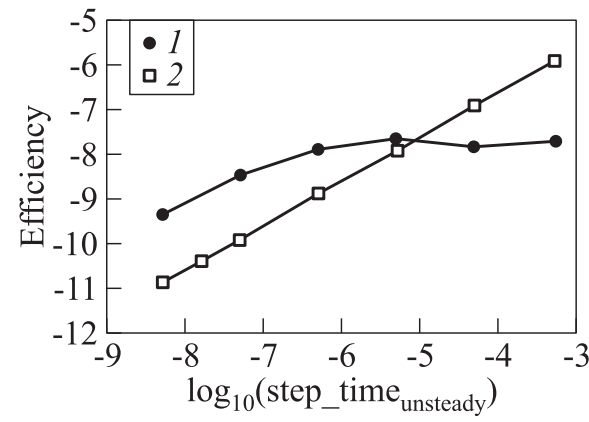

Figure 15 Computational efficiency of zonal approach (1) for wing: $2-$ dual time stepping

\section{CONCLUDING REMARKS}

1. Calculations of flow around the RA16SC1 three-element airfoil at different incidence angles for regime $\mathrm{M}=0.15$ and $\mathrm{Re}_{c}=1.7 \cdot 10^{6}$ are in a good correspondence with the experimental data obtained in Low Speed Wind Tunnel of Airbus Bremen (Germany) in the framework of EUROPIV2 Project. But the flap upper surface flow separation obtained by computation disappears earlier than that one obtained by experiment. One may suppose that the calculation overestimates the turbulence intensity in the boundary layer at the airfoil main element upper surface and in the following mixing layer. The stall is generated at the airfoil main element upper surface for $\alpha \approx 24^{\circ}$.

2. Dependences $C_{p x}(t)$ and $C_{p y}(t)$, which are obtained in the calculations of airfoil RA16SC1, confirm initial assumption that the calculations of such class flows can be performed with large Courant numbers at the depth of boundary layers, if the condition CFL $\sim 1$ is satisfied in the outer part of the boundary layer and in the zone of inviscid flow around the airfoil.

3. When the conditions described in the conclusion 2 are satisfied, the proposed zonal approach permits to accelerate the description of nonstationary processes in flow around RA16SC1 three-element airfoil more than 10 times in comparison with the standard approach based on using the implicit scheme with dual-time step.

4. The calculations of flow around the TC217 model (combination "fuselageKH3Y wing") at different incidence angles for regime $\mathrm{M}=0.2$ and 
$\operatorname{Re}_{c}=15 \cdot 10^{6}$ using the implicit scheme with dual-time step for regimes without flow separation are in good correspondence with the experimental data obtained in ETW (Cologne, Germany) in the framework of the EUROLIFT II Project. To achieve the desirable accuracy of flow simulation, it is sufficiently to use the grid "mesh1" (2 million cells). Both in the experiment and in the calculations for grids "mesh2" and "mesh1," the flow separation begins at the wing tip; the flow separates from the slat upper surface.

5. It is shown that the Courant number in the boundary layer outer part and in inviscid flow zone around the airfoil influences strongly the prediction of flow separation appearance from the wing upper surface. It was succeeded to predict the flow separation appearance with the satisfactory accuracy only for the coarsest grid - "mesh2," with CFL 100 in ROI. As the Courant number growing, the appearance moment, at first, is delayed and then begins to evolve nonphysically.

6. To obtain the total polar $C_{L}\left(C_{D}\right)$, which is similar to the experimental data, one should recommend performing the calculation for the grid "mesh1" but using the global time step, which provides the condition CFL $\sim 1$ in ROI. The zonal approach in this case is efficient more than 2 times than the calculation using the implicit scheme with dual-time step.

\section{REFERENCES}

1. Bosnyakov, S., I. Kursakov, A. Lysenkov, S. Matyash, S. Mikhailov, V. Vlasenko, and J. Quest. 2008. Computational tools for supporting the testing of civil aircraft configurations in wind tunnels. Prog. Aerosp. Sci. 44(2):67-120.

2. Pervaiz, M. M., and J.R. Baron. 1989. Spatiotemporal adaptation algorithm for two-dimensional reacting flows. AIAA J. 27(10):1368-1376.

3. Holst, T. L. 1987. Viscous Transonic Airfoil Workshop compendium of results. AIAA Paper No. 87-1460.

4. Neitzke, K. P. 2002. Data evaluation and discussion of the lift coefficient. EUROPIV2 Workshop.

5. Wild, J., J. Brezillon, O. Amoignon, J. Quest, F. Moens, and D. Quagliarella. 2007. Advanced high-lift design by numerical methods and wind tunnel verification within the European Project EUROLIFT II. AIAA Paper No. 2007-4300.

6. Eliasson, P. 2008. Investigation of a half-model high-lift configuration in a wind tunnel. J. Aircraft 45(1):29-37. 